\title{
Outcomes of Truncus Arteriosus Repair and Predictors with Mortality
}

\author{
Mohammed Hamzah ${ }^{1}$, Hasan F. Othman ${ }^{2}$, Kshama Daphtary ${ }^{1}$, Rukmini Komarlu ${ }^{1}$, and \\ Hany Aly ${ }^{1}$ \\ ${ }^{1}$ Cleveland Clinic Children's Hospital \\ ${ }^{2}$ Sparrow Health System
}

April 28, 2020

\begin{abstract}
Abstract: The objective of this study was to identify patient and hospitalization characteristics associated with in-hospital mortality in infants with truncus arteriosus. We conducted a retrospective analysis of a large administrative database, the National Inpatient Sample dataset of the Healthcare Cost and Utilization Project for the years 2002-2017. We also sought to evaluate the resource utilization in the subgroup of subjects with truncus arteriosus and 22q11.2 deletion syndrome. Neonates with truncus arteriosus were identified by ICD-9 and ICD-10 codes. Hospital and patient factors associated with inpatient mortality were analyzed. Overall, 3009 neonates met inclusion criteria; a total of 326 patients died during the hospitalization (10.8\%). Extracorporeal membrane oxygenation utilization was $7.1 \%$. Univariate and multivariate logistic regression analyses were used to identify risk factors for in-hospital mortality. Independent risk factors for mortality were prematurity $(\mathrm{aOR}=$ 2.43, 95\% CI: $1.40-4.22, \mathrm{p}=0.002)$, diagnosis of stroke $(\mathrm{aOR}=26.2,95 \% \mathrm{CI}: 10.1-68.1, \mathrm{p}<0.001)$, necrotizing enterocolitis $(\mathrm{aOR}=3.10,95 \% \mathrm{CI}: 1.24-7.74, \mathrm{p}=0.015)$ and presence of venous thrombosis $(\mathrm{aOR}=13.5,95 \% \mathrm{CI}: 6.7-27.2, \mathrm{p}<0.001)$. Patients who received ECMO support or had cardiac catheterization procedure during the hospitalization had increased odds of mortality $(\mathrm{aOR}=82.0,95 \% \mathrm{CI}: 44.5-151.4, \mathrm{p}<0.001$, and $\mathrm{aOR}=1.65,95 \%$ CI: $0.98-2.77, \mathrm{p}=0.060$, respectively). $22 \mathrm{q} 11.2$ deletion syndrome was associated with an inverse risk of death despite having more non-cardiac comorbidities; this patient subpopulation also had higher length of stay and increased cost of hospitalization.
\end{abstract}

\section{Title:}

Outcomes of Truncus Arteriosus Repair and Predictors with Mortality

\section{Authors:}

Mohammed Hamzah, MD ${ }^{\mathrm{a}}$; Hasan F. Othman, MD ${ }^{\mathrm{b}}$; Kshama Daphtary, MD ${ }^{\mathrm{a}}$; Rukmini Komarlu, MD Hany Aly, MD

\section{Affiliations:}

a Department of Pediatric Critical Care, Cleveland Clinic Children's, Cleveland, OH

b Department of Pediatrics, Michigan State University/Sparrow Health System, Lansing, MI

c Department of Pediatric Cardiology, Cleveland Clinic Children's, Cleveland, OH

d Department of Neonatology, Cleveland Clinic Children's, Cleveland, OH 
Address correspondence to: Mohammed Hamzah, Department of Pediatric Critical Care, Cleveland Clinic Children's,

9500 Euclid Ave. M14, Cleveland, OH 44195, E-mail: HamzahM@ccf.org, Fax: (216) 444-3310, Telephone: (216) 444-0732

Short title: Outcomes of truncus arteriosus in children.

Funding Source: No funding was secured for this study.

Financial Disclosure: All authors have no financial relationships relevant to this article to disclose.

Conflict of Interest: All authors have no conflicts of interest to disclose.

\section{Abbreviations:}

NIS: National Inpatient Sample

HCUP: Healthcare Cost and Utilization Project

AHRQ: Agency for Healthcare Research and Quality

ICD-9: International Classification of Disease-Ninths edition

ICD-10: International Classification of Disease-Tenths edition

LOS: length of stay

OR: odds ratio

IQR: Inter Quartile Range.

CI: confidence interval

ECMO: Extra Corporeal Membrane Oxygenation

AKI: Acute Kidney Injury

Keywords: truncus arteriosus; outcomes; ECMO; 22q11.2 deletion syndrome

\section{Contributor's Statement Page:}

Dr. Hamzah conceptualized and designed the study, drafted and revised the manuscript.

Dr. Othman carried out the analyses and reviewed and revised the manuscript.

Dr. Daphtary contributed to the initial draft of the manuscript and revised the manuscript.

Dr. Komarlu contributed to the initial draft of the manuscript and revised the manuscript.

Dr. Aly designed the study, critically reviewed the manuscript for important intellectual content.

All authors approved the final manuscript as submitted and agree to be accountable for all aspects of the work.

\section{Abstract:}

The objective of this study was to identify patient and hospitalization characteristics associated with inhospital mortality in infants with truncus arteriosus. We conducted a retrospective analysis of a large administrative database, the National Inpatient Sample dataset of the Healthcare Cost and Utilization Project for the years 2002-2017. We also sought to evaluate the resource utilization in the subgroup of subjects with truncus arteriosus and 22q11.2 deletion syndrome. Neonates with truncus arteriosus were identified by ICD-9 and ICD-10 codes. Hospital and patient factors associated with inpatient mortality were 
analyzed. Overall, 3009 neonates met inclusion criteria; a total of 326 patients died during the hospitalization (10.8\%). Extracorporeal membrane oxygenation utilization was $7.1 \%$. Univariate and multivariate logistic regression analyses were used to identify risk factors for in-hospital mortality. Independent risk factors for mortality were prematurity $(\mathrm{aOR}=2.43,95 \%$ CI: $1.40-4.22, \mathrm{p}=0.002)$, diagnosis of stroke $(\mathrm{aOR}=$ 26.2, 95\% CI: 10.1-68.1, $\mathrm{p}<0.001)$, necrotizing enterocolitis $(\mathrm{aOR}=3.10,95 \%$ CI: $1.24-7.74, \mathrm{p}=0.015)$ and presence of venous thrombosis $(\mathrm{aOR}=13.5,95 \%$ CI: $6.7-27.2, \mathrm{p}<0.001)$. Patients who received ECMO support or had cardiac catheterization procedure during the hospitalization had increased odds of mortality $(\mathrm{aOR}=82.0,95 \%$ CI: 44.5-151.4, $\mathrm{p}<0.001$, and $\mathrm{aOR}=1.65,95 \%$ CI: $0.98-2.77, \mathrm{p}=0.060$, respectively). 22q11.2 deletion syndrome was associated with an inverse risk of death despite having more non-cardiac comorbidities; this patient subpopulation also had higher length of stay and increased cost of hospitalization.

\section{Introduction:}

Truncus arteriosus is an infrequent cyanotic congenital heart disease that occurs at a rate of $0.56-1.3$ per 10,000 live births (1). Surgical mortality in patients with truncus arteriosus is high relative to other congenital heart disease and is reported to be between $6.9-11 \%(2,3)$. Few studies had evaluated outcomes and risk factors for mortality in truncus arteriosus; these studies were limited by either small sample size or single center studies (4-6). The main objective of this study was to identify patient and hospitalization characteristics associated with mortality in infants with truncus arteriosus who had surgical repair. We evaluated 3,009 infants who underwent truncus arteriosus surgical repair. This is the largest cohort of patients with repaired truncus arteriosus. We hypothesized that using data from a large multicenter national database with both a large number of patients and a wide range of practice variations would allow us to evaluate the surgical outcomes better and to identify the factors associated with mortality in infants who had truncus arteriosus repair. We also sought to evaluate the resource utilization in the subgroup of subjects with truncus arteriosus and 22q11.2 deletion syndrome. Syndrome of 22q11.2 deletion occurs in $25 \%$ of patients with truncus arteriosus. We hypothesize that having $22 \mathrm{q} 11.2$ deletion syndrome would influence the resource utilization in the postoperative period, as these subjects would have a prolonged and more complicated postoperative course given the presence of immunodeficiency and multiple non-cardiac anomalies that are associated with 22q11.2 deletion syndrome (7).

\section{Methods}

The study was conducted using de-identified patient data from the National Inpatient Sample (NIS) database, a part of the Healthcare Cost and Utilization Project (HCUP), which is compiled by the agency for Health care Research and Quality (AHRQ). The NIS database randomly samples 20 percent of the discharges from participating hospitals in 47 US states and the District of Columbia. It contains data from more than seven million hospital stays each year (8). Data from the years 2000-2017 were used for the purposes of this study. The study was exempt from an institutional board review as the use of data from approved unidentified public data sets is not considered human subject research.

The study population was identified using the International Classification of Disease, Ninth and Tenth Revisions, Clinical Modification (ICD-9\&10-CM). Patients were included in the study if they meet the following criteria. In essence, the patient was less than one year of age, had a diagnosis of truncus arteriosus (ICD codes: 745.0 \& Q20.0), and had truncus arteriosus repair procedure (ICD-9-PCS: 35.83,35.92 and corresponding ICD-10 procedure codes) during the index of hospitalization. Patient data queried included demographics and patient clinical characteristics. Patient demographic data collected included: age, sex, race, primary payer, location of the hospital, type of hospital, hospital bed size, the region of the hospital, type of admission (elective or non-elective) and admission day of the week (weekend or weekday). Primary outcomes were identified before the analysis; these included death before hospital discharge, length of stay (LOS), and hospital cost of charge. We compiled a list of common procedures, comorbidities, and complications and evaluated their prevalence in those who died during the hospitalization. For the analysis of outcomes in patients 
with 22q11.2 deletion syndrome, patients were stratified into two groups: those with and without 22q11.2 deletion syndrome using ICD code 279.11, 758.32, D82.1, and Q93.81. Data were described using median (interquartile range) for continuous variables as deemed appropriate. Categorical variables were described as frequency (percentage). Survivors and Non-survivors were compared with regards to demographic, clinical characteristics, and outcomes using Mann-Whitney U test for continuous characteristics and Chi-square or Fisher's exact tests for categorical characteristics. Frequency of truncus arteriosus repair, in-hospital mortality, and LOS were evaluated over the years of the study. Trends were assessed for significance using Jonckheere-Terpstra test. $\mathrm{P}<0.05$ was considered significant. In an effort to minimize bias and to adjust for measurable confounders, a univariate analysis was initially performed to identify potential predictors of in-hospital mortality, thereafter, variables with statistical significance $(\mathrm{p}<0.15)$ were incorporated in a multivariable analysis using a logistic regression model. We used the SPSS software, version 25 (SPSS Inc., Chicago, IL) for all statistical analyses.

\section{Results:}

Overall, there were 3009 infants with truncus arteriosus that met the inclusion criteria. The majority of these patients $(2026,67.4 \%)$ had their surgical repair at [?] 28 days of age. There was no predominance of either sex (male $51.2 \%$ vs. female $48.8 \%$ ). The majority of the patients were of the White race $(51.6 \%$ ), followed by the Hispanic race $(23.3 \%)$. Primary payer insurance was equally divided between Private insurance and Medicaid (46.6\% and $46.3 \%$, respectively). The majority of the patients were admitted to large bed-size hospitals (59.9\%). Patients discharged from the hospital were equally spread throughout the year with no seasonal variation. The majority of the hospitalizations were non-elective admissions (78.8\%). In-hospital mortality was $326(10.8 \%)$. Mortality was stable with a non-significant decrease over the years of the study (Std. J-T statistics $-1.063, \mathrm{p}=0.288$, Figure 1). Characteristics of survivors versus non-survivors are presented in table 1. Mortality rates were higher in neonates $(12.9 \%$ vs. $6.4 \%, \mathrm{p}<0.001)$. Patients with Black, Native American or Hispanic race had significantly higher mortality than White race $(16.5 \%, 15.6 \%$, $13.5 \%$ vs. $7.9 \%, \mathrm{p}<0.001$, respectively) and mortality in patients with Medicaid insurance was significantly higher than those with private insurance $(14.4 \%$ vs. $8.0 \%, \mathrm{p}<0.001)$. Mortality rates during elective admissions was $7.4 \%$ versus $10.6 \%$ for non-elective admissions $(\mathrm{p}=0.020)$.

Table 2 shows the outcomes of infants with repaired truncus arteriosus. Non-survivors had prolonged median length of stay (non-survivors 29 days [IQR 12-61] vs. survivors 24 days [IQR 14-40], p = 0.009), this was associated with twofold increase in the cost of hospitalization in the non-survivors (non-survivors $\$ 522,615[\$ 253,366-\$ 842,009]$ vs. survivors $\$ 285,114[\$ 170,681-\$ 526,175], \mathrm{p}<0.001)$. Of note, $47.4 \%$ of the non-survivors were receiving ExtraCorporeal Membrane Oxygenation (ECMO) life support during the hospitalization versus $2.2 \%$ of survivors $(\mathrm{OR}=39.4, \mathrm{p}<0.001)$. Prematurity (gestational age $<37$ weeks) and low birth weight (weight $<2500 \mathrm{~g}$ ) were associated with increased odds risk of mortality $(\mathrm{OR}=1.95,95 \%$ CI: 1.40-2.72, $\mathrm{p}<0.001$, and $\mathrm{OR}=1.39,95 \%$ CI: $0.95-2.03, \mathrm{p}=0.087$, respectively). Chromosomal anomalies other than 22q11.2 deletion syndrome were present at a similar frequency in both survivors and non-survivors (survivors $4.7 \%$ vs. non-survivors $4.9 \%, \mathrm{p}=0.782$ ). Surprisingly, 22q11.2 deletion syndrome was present less frequently in non-survivors (non-survivors $20.9 \%$ versus survivors $28.0 \%, \mathrm{p}=0.007$ ). Overall, 22q11.2 deletion syndrome was present in $27.2 \%$ of the patients. In table 3, we compiled a list of in-hospital complications and evaluated the association of these complications with in-hospital mortality (table 2). Stroke, acute kidney injury (AKI), pleural effusion, venous thrombosis, intraventricular hemorrhage and necrotizing enterocolitis were all associated with increased odds risk of mortality $(\mathrm{OR}=12.8,4.37,1.69,4.85,1.73$ and 4.89 , respectively). Pulmonary hypertension was not associated with an increased odds risk of mortality.

A univariate analysis followed by a multivariable regression analysis was performed to further analyze the dynamics between mortality predictors (tables 3 and 4 ). The following factors were found to be associated with increased mortality in the multivariate analysis: prematurity $(\mathrm{aOR}=2.43,95 \%$ CI: $1.40-4.22, \mathrm{p}=$ $0.002)$, diagnosis of stroke $(\mathrm{aOR}=26.2,95 \% \mathrm{CI}: 10.1-68.1, \mathrm{p}<0.001)$, necrotizing enterocolitis $(\mathrm{aOR}=$ 3.10, 95\% CI: $1.24-7.74, \mathrm{p}=0.015)$ and presence of venous thrombosis $(\mathrm{aOR}=13.5,95 \%$ CI: $6.7-27.2, \mathrm{p}<$ 0.001). Private insurance and 22q11.2 deletion syndrome were associated with lower odds of mortality (aOR 
$=0.34,95 \%$ CI: $0.20-0.55, \mathrm{p}<0.001$, and $\mathrm{aOR}=0.54,95 \%$ CI: $0.34-0.87, \mathrm{p}=0.011$, respectively). In the multivariate analyses: AKI and pleural effusion were not associated with increased mortality. Patients who received ECMO support or had cardiac catheterization procedure during the hospitalization had increased odds of mortality $(\mathrm{aOR}=82.0,95 \%$ CI: 44.5-151.4, $\mathrm{p}<0.001$, and $\mathrm{aOR}=1.65,95 \%$ CI: $0.98-2.77, \mathrm{p}=$ 0.060 , respectively). Successful cardiopulmonary resuscitation during the hospitalization was not associated with a significant increase in mortality (aOR $=1.29,95 \% \mathrm{CI}: 0.54-3.11, \mathrm{P}=0.570)$.

\section{Outcomes of the subgroup with 22q11.2 deletion syndrome (Table 5):}

22q11.2 deletion syndrome was present in $820(27.2 \%)$ patients with truncus arteriosus who had a surgical repair. Patients with 22q11.2 deletion syndrome had significantly prolonged median length of stay (32 days [IQR: 19-53] compared to 22 days [IQR: 12-37], $\mathrm{p}<0.001)$ and this was associated with significant increase in median cost of hospitalization ( $\$ 382,621$ [IQR $\$ 231,360-\$ 657,527$ ] versus $\$ 273,713$ [IQR $\$ 163,272$ $\$ 522,615], \mathrm{p}<0.001)$. Lower mortality rates were observed in the $22 \mathrm{q} 11.2$ deletion syndrome group $(8.3 \%$ versus $11.7 \%, \mathrm{p}=0.007)$. The two groups $(22 \mathrm{q} 11.2$ deletion and no-22q11.2 deletion) had similar baseline characteristics and comorbidities. Low birth weight, prematurity (gestational age $<37$ weeks) were present in similar frequency in both groups. Similarly, ECMO, cardiopulmonary resuscitation, mechanical ventilation, and cardiac catheterization procedures were utilized at a similar rate in both groups.

Of significance, the study finds that patients with 22q11.2 deletion syndrome had a higher incidence of noncardiac anomalies. More airway anomalies were present in the 22q11.2 deletion group (10.9\% vs. 5.9\%, OR $=1.94,95 \%$ CI: $1.47-2.58, \mathrm{p}<0.001)$, chromosomal anomalies other than 22q11.2 deletion were present at higher frequency $(7.3 \%$ vs. $3.7 \%, \mathrm{p}<0.001)$. In association with these comorbidities, we observed that tracheostomy and G-tube placement were performed more frequently in the 22q11.2 deletion group $(5.4 \%$ vs. $2.7 \%, \mathrm{OR}=2.05,95 \%$ CI: $1.37-3.05, \mathrm{p}<0.001$, and $12.9 \%$ vs. $4.5 \%, \mathrm{OR}=3.13,95 \%$ CI: $2.35-4.18, \mathrm{p}$ $=0.001$, respectively). Kidney anomalies, acute kidney injury, venous thrombosis, necrotizing enterocolitis, and pulmonary hypertension were present in similar frequency in both groups (Table 5).

\section{Discussion:}

Using a large multicenter national database, we reported the outcomes of 3,009 infants with truncus arteriosus who underwent surgical repair. The study provides descriptive but relevant information with regards to demographics, national trends, in-hospital complications, and outcomes in infants with truncus arteriosus. The overall mortality in truncus arteriosus is $10.8 \%$. A recent report from the society of thoracic surgeons congenital heart surgery database showed a similar mortality rate of $9.6 \%$ in truncus arteriosus repair, which is the second-highest rate of mortality after Norwood procedure (9). Aiming to identify predictors of mortality in this disease, a multivariate regression analysis was used, and we also analyzed the outcomes in subjects with 22q11.2 deletion syndrome. The main findings in this study were as follows: (1) Patient characteristics that were associated with mortality were a non-White race, prematurity, and Medicaid insurance. (2) 22q11.2 deletion syndrome was associated with an inverse risk of death despite having more non-cardiac comorbidities. (3) 22q11.2 deletion syndrome patients had a longer length of hospital stay and higher cost of hospitalization.

To address the first finding of insurance disparities, Erickson and colleagues showed in their study that infants with congenital heart disease and private insurance are cared for at hospitals with lower surgical mortality and that non-White children with Medicaid insurance were less likely to use a hospital with lower mortality rate (10). In addition, prematurity carries a major risk factor for increased mortality in infants with congenital heart disease, and preterm birth rates vary by race and ethnicity. Socioeconomic and ethnic-racial disparities are a major risk for prematurity (11). Studies have shown that non-Hispanic Black women are 2.5 times more likely to have preterm birth versus White women (12). The dynamics and interplay between these three risk factors (preterm birth, non-White race, and government insurance) are challenging, and the coexistence of these factors is not uncommon. The second main finding in this study was the presence of an inverse 
risk of death associated with 22q11.2 deletion syndrome. The baseline characteristics for these patients are similar to subjects without -22q11.2 deletion syndrome. Many researchers have examined the operative mortality in truncus arteriosus and some have found that 22q11.2 deletion syndrome does not necessarily increase surgical risk (13-15), while others reveal that 22q11.2 deletion is an independent risk factor for late mortality $(16,17)$. However, the results of our study are in agreement with the results of a study by Michielon and colleagues, which found that 22q11.2 deletion syndrome was an inverse risk for death in patients with truncus arteriosus (18). It could also be that the complex cardiac pathology and challenging nature of the repair with its prolonged postoperative course could have much more of an impact than the impact of the diagnosis of 22q11.2 deletion on truncus arteriosus. , One could also hypothesize that the cardiac morphology and anatomy in these patients is different from patients without 22q11.2 deletion syndrome. A study by McElhinney reviewed details of cardiac features in truncus patients with 22q11.2 deletion and showed that patients with 22q11.2 deletion had a higher frequency of right-sided aortic arch and abnormal aortic arch branching. Most importantly, interrupted aortic arch, which could impact operative mortality, occurred at a similar frequency in those with and without 22q11.2 deletion syndrome. In McElhinney's study, there was a statistically insignificant trend for a lower incidence of moderate-severe truncal valve insufficiency in patients with 22q11.2 deletion (11\% vs. 33\%) which could impact surgical mortality, suggesting that factors other than chromosome 22q11.2 deletion are the main determinants of the functional and morphological characteristics of the truncal valve (19). Previous studies have shown the association with poor surgical outcomes in subjects with truncal valve regurgitation, interrupted aortic arch and coronary artery anomalies (20). One of the limitations of our study is the inability to review patient charts and cardiac imaging to evaluate for the presence of postoperative truncal valve regurgitation or the details of coronary anatomy in these patients. Future studies that also evaluate the cardiac anatomy in 22q11.2 deletion syndrome will likely be able to evaluate our finding of an inverse death risk that is associated with 22q11.2 syndrome in truncus arteriosus.

Lastly, this study reported prolonged hospitalization and increased cost of hospitalization in patients with truncus arteriosus. This is similar to the study done by Ghimire et al, who showed that the diagnosis of 22q11.1 deletion is associated with longer hospital stay and higher hospitalization cost (21). The 22q11.2 group had far more non-cardiac comorbidities and had higher rates of postoperative complications. Feeding issues were more common in 22q11.2 deletion syndrome, a higher percentage of these patients had a gastrostomy tube during the index hospitalization. Airway anomalies were present more frequently in 22q11.2 deletion, and this was associated with a higher frequency of tracheostomy procedure. Immunological dysfunction is not uncommon in infants with 22q11.2 deletion (22), and it was not surprising to find higher rates of septicemia and fungal infection in the 22q11.2 deletion group. Therefore, it was not unexpected to have a prolonged postoperative hospitalization in patients with 22q11.2 deletion, modifications in the perioperative care of this subpopulation to address these complications is warranted to further shorten postoperative course and decrease cost of hospitalization.

Limitations: There are several limitations to this study, the study relied on the ICD-9 \& ICD-10 codes for the diagnosis of truncus arteriosus and 22q11.2 deletion syndrome, it didn't describe how the chromosomal deletion diagnosis was reached. The study span is over a span of two decades; the field of molecular genetic testing and genomic profiling has evolved dramatically; this could have affected the accuracy of the genetic screening method over the years of the study. The database does not provide details of cardiac anatomy, mainly coronary artery anomalies, postoperative truncal valve insufficiency and aortic arch obstruction. The database lacks information on cardiac bypass and surgical procedure techniques, which have evolved over the course of these two decades and therefore is subject to an era effect. These factors would impact the surgical outcomes in truncus arteriosus. The current study benefited from the large sample size, some limitations are inherent to all retrospective studies using administrative databases. Incorrect or missing data may exist. We rely mainly on ICD diagnostic and procedure codes to gather information, and we were not able to refer to specific patient hospital charts to determine the cause of death. The lack of validation of the data collected by chart review is a source of potential bias for errors. However, in a large study such as ours, the patients' volume likely offset these inaccuracies. Another limitation of our study is the restriction to the 
hospitalization period, and this prevented us from evaluating long-term outcomes.

\section{Conclusion:}

Truncus arteriosus is an infrequent congenital heart disease with mortality comparable to that following Norwood procedure. Mortality rates have trended down over time although continue to remain high. 22q11.2 deletion was not associated with increased mortality compared to subjects without this deletion. However this subject population had higher incidence of non-cardiac anomalies, higher need for tracheostomy and G-tube placement, protracted length of stay with increased cost of hospitalization. Prematurity, non-White race and use of Medicaid insurance were factors associated with higher mortality. These findings will help direct prenatal counseling and management postnatally.

\section{References:}

1. Ferencz C, Rubin JD, Mccarter RJ, et al.: Congenital heart disease : Prevalence at livebirth: The Baltimore-Washington infant study. Am J Epidemiol 1985; 121:31-36

2. Mastropietro CW, Amula V, Sassalos P, et al.: Characteristics and operative outcomes for children undergoing repair of truncus arteriosus: A contemporary multicenter analysis [Internet].J Thorac Cardiovasc Surg 2019; 157:2386-2398.e4Available from:https://doi.org/10.1016/j.jtcvs.2018.12.115

3. Russell HM, Pasquali SK, Jacobs JP, et al.: Outcomes of repair of common arterial trunk with truncal valve surgery: A review of the society of thoracic surgeons congenital heart surgery database [Internet]. Ann Thorac Surg 2012; 93:164-169Available from:http://dx.doi.org/10.1016/j.athoracsur.2011.04.121

4. Morgan CT, Tang A, Fan CP, et al.: Contemporary Outcomes and Factors Associated With Mortality After a Fetal or Postnatal Diagnosis of Common Arterial Trunk [Internet]. Can J Cardiol 2019; 35:446-452Available from: https://doi.org/10.1016/j.cjca.2018.12.006

5. Naimo PS, Fricke TA, Yong MS, et al.: Outcomes of Truncus Arteriosus Repair in Children: 35 Years of Experience From a Single Institution [Internet]. Semin Thorac Cardiovasc Surg 2016; 28:500511Available from: http://dx.doi.org/10.1053/j.semtcvs.2015.08.009

6. Brown JW, Ruzmetov M, Okada Y, et al.: Truncus arteriosus repair: Outcomes, risk factors, reoperation and management. Eur J Cardio-thoracic Surg 2001; 20:221-227

7. Crowley B, Ruffner M, McDonald McGinn DM, et al.: Variable immune deficiency related to deletion size in chromosome 22q11.2 deletion syndrome. Am J Med Genet Part A 2018; 176:2082-2086

8. Agency for Healthcare Research and Quality. Overview of the National (Nationwide) Inpatient Sample (NIS). Available from: https://www.hcup-us.ahrq.gov/nisoverview.jsp Accessed February 20, 2020.

9. Jacobs JP, Mayer JE, Pasquali SK, et al.: The Society of Thoracic Surgeons Congenital Heart Surgery Database: 2019 Update on Outcomes and Quality [Internet]. Ann Thorac Surg 2019; 107:691704Available from:https://doi.org/10.1016/j.athoracsur.2018.12.016

10. Erickson LC, Wise PH, Cook EF, et al.: The Impact of Managed Care Insurance on Use of LowerMortality Hospitals by Children Undergoing Cardiac Surgery in California. Pediatrics 2004; 105:12711278

11. Kramer MR, Hogue CJ, Dunlop AL, Menon R. Preconceptional stress and racial disparities in preterm birth: an overview. Acta Obstet Gynecol Scand. 2011;90(12):1307-1316.

12. Thoma ME, Drew LB, Hirai AH, et al.: Black-White Disparities in Preterm Birth: Geographic, Social, and Health Determinants [Internet]. Am J Prev Med 2019; 57:675-686Available from:https://doi.org/10.1016/j.amepre.

13. Alsoufi B, McCracken C, Shashidharan S, et al.: The Impact of 22q11.2 Deletion Syndrome on Surgical Repair Outcomes of Conotruncal Cardiac Anomalies [Internet]. Ann Thorac Surg 2017; 104:15971604Available from: https://doi.org/10.1016/j.athoracsur.2017.04.019

14. Cuturilo G, Drakulic D, Jovanovic I, et al.: The Impact of 22q11.2 Microdeletion on Cardiac Surgery Postoperative Outcome. Pediatr Cardiol 2017; 38:1680-1685 
15. O’Byrne ML, Yang W, Mercer-Rosa L, et al.: 22q11.2 Deletion syndrome is associated with increased perioperative events and more complicated postoperative course in infants undergoing infant operative correction of truncus arteriosus communis or interrupted aortic arch [Internet]. J Thorac Cardiovasc Surg 2014; 148:1597-1605Available from:http://dx.doi.org/10.1016/j.jtcvs.2014.02.011

16. Buckley $\mathrm{JR}^{1}$, Amula $\mathrm{V}^{2}$, Sassalos $\mathrm{P}^{3}$, Costello $\mathrm{JM}^{4}$, Smerling $\mathrm{AJ}^{5}$, Iliopoulos $\mathrm{I}^{6}$, Jennings $\mathrm{A}^{7}$, Riley $\mathrm{CM}^{8}$, Cashen $\mathrm{K}^{9}$, Suguna Narasimhulu $\mathrm{S}^{10}$, Gowda $\mathrm{KMN}^{11}$, Bakar $\mathrm{AM}^{12}$, Wilhelm $\mathrm{M}^{13}$, Badheka $\mathrm{A}^{14}$, Moser EAS ${ }^{15}$, Mastropietro $\mathrm{CW}^{16}$; Collaborative Research in Pediatric Cardiac Intensive Care Investigators. Multicenter analysis of early childhood outcomes after repair of truncus arteriosus. Ann Thorac Surg . 2019 Feb;107(2):553-559. doi: 10.1016/j.athoracsur.2018.08.094. Epub 2018 Oct 26.

17. Naimo $\mathrm{PS}^{1}$, Fricke $\mathrm{TA}^{1}$, Yong $\mathrm{MS}^{2}$, d'Udekem $\mathrm{Y}^{3}$, Kelly $\mathrm{A}^{4}$, Radford DJ ${ }^{5}$, Bullock $\mathrm{A}^{6}$, Weintraub $\mathrm{RG}^{3}$, Brizard $\mathrm{CP}^{3}$, Konstantinov $\mathrm{IE}^{7}$. Outcomes of truncus arteriosus repair in children: 35 years of experience from a single institution.Semin Thorac Cardiovasc Surg. 2016 Summer; 28(2):500-511. doi: 10.1053/j.semtcvs.2015.08.009.

18. Michielon G, Marino B, Oricchio G, et al.: Impact of DEL22q11, trisomy 21, and other genetic syndromes on surgical outcome of conotruncal heart defects [Internet]. J Thorac Cardiovasc Surg 2009; 138:565-570.e2Available from: http://dx.doi.org/10.1016/j.jtcvs.2009.03.009

19. McElhinney DB, Driscoll DA, Emanuel BS, et al.: Chromosome 22q11 Deletion in Patients with Truncus Arteriosus. Pediatr Cardiol2003; 24:569-573

20. Konstantinov IE, Karamlou T, Blackstone EH, et al.: Truncus arteriosus associated with interrupted aortic arch in 50 neonates: A congenital heart surgeons society study. Ann Thorac Surg 2006; 81:214222.

21. Ghimire LV, Devoe C'Moon-Grady AJ 22q11.2 deletion status influences resource utilization in infants requiring repair of Tetralogy of Fallot and common arterial trunk. Pediatr Cardiol.2020 Feb 28. doi: 10.1007/s00246-020-02333-y. [Epub ahead of print]

22. Morsheimer M, Brown Whitehorn TF, Heimall J, et al.: The immune deficiency of chromosome 22q11.2 deletion syndrome. Am J Med Genet Part A 2017; 173:2366-2372

\section{Hosted file}

Table 1.docx available at https://authorea.com/users/311858/articles/442495-outcomes-of-truncusarteriosus-repair-and-predictors-with-mortality

\section{Hosted file}

Table 2.docx available at https://authorea.com/users/311858/articles/442495-outcomes-of-truncusarteriosus-repair-and-predictors-with-mortality

\section{Hosted file}

Table 3.docx available at https://authorea.com/users/311858/articles/442495-outcomes-of-truncusarteriosus-repair-and-predictors-with-mortality

\section{Hosted file}

Table 4.docx available at https://authorea.com/users/311858/articles/442495-outcomes-of-truncusarteriosus-repair-and-predictors-with-mortality

\section{Hosted file}

Table 5.docx available at https://authorea.com/users/311858/articles/442495-outcomes-of-truncusarteriosus-repair-and-predictors-with-mortality 


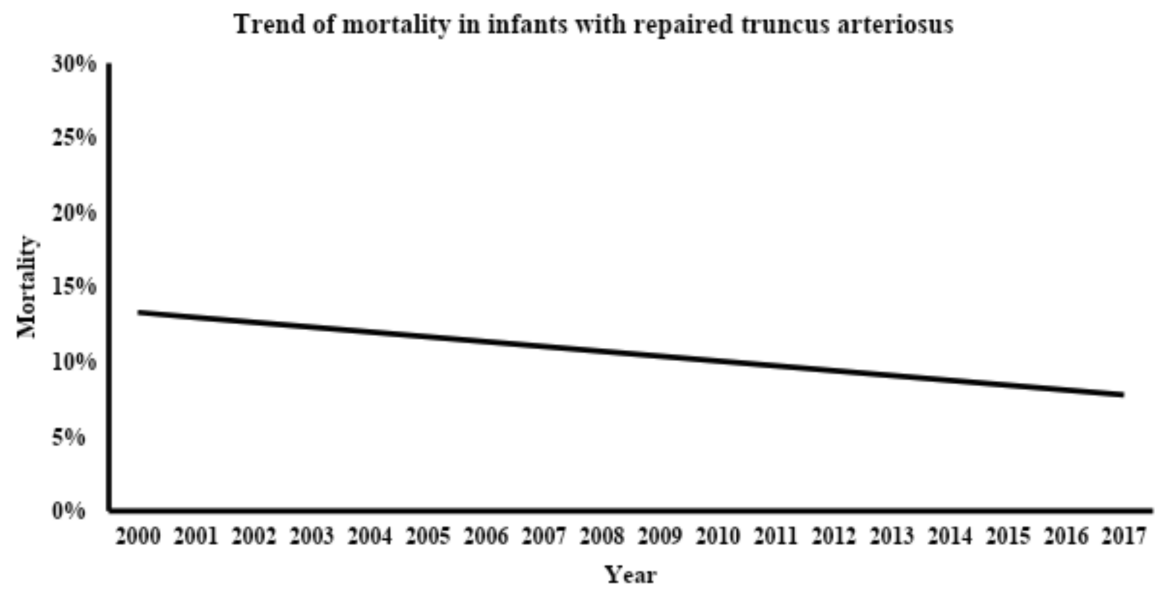

